\title{
Die dunkle Seite der Vorstellungskraft: Das Abjekt als Verworfenes im Imaginären
}

\author{
Larissa Pfaller
}

Angenommen: 1. April 2021 / Online publiziert: 27. April 2021

(C) Der/die Autor(en) 2021

Zusammenfassung Die sich derzeitig konstituierende Soziologie des Imaginären nimmt dieses in erster Linie über die Rekonstruktion bedeutungstragender Vorstellungsinhalte in den Blick. Demgegenüber bleibt dasjenige, was im Imaginären verworfen wird, außerhalb empirischer Forschung wie theoretischer Betrachtungen. Um diese Lücke zu schließen, schlägt der Artikel den Begriff des Abjekts vor, dessen analytisches Potenzial für die Soziologie bisher ungenutzt bleibt. Am Beispiel des Phänomens des vierten Alters wird anschließend vorgeführt, wie eine Analyse des Abjekts systematisch für die Rekonstruktion des sozialen Imaginären nutzbar gemacht werden kann. Im Sinne einer Soziologie des Exzeptionellen plädiert der Artikel abschließend dafür, auch das soziale Imaginäre stets von seinen Grenzen aus zu denken.

Schlüsselwörter Soziales Imaginäres · Imagination · Abjekt · Abjektion · Viertes Alter 


\section{The dark side of imagination: abjection and the social imaginary}

Abstract There is a new growing interest in imagination and social imaginaries in sociology. Existing approaches analyze social imaginaries as sets of cultural conceptions that guide human actions and understandings of the world. However, the dismissed, discarded and abjected is barely part of empirical research and theoretical considerations. To address this desideratum, the article introduces the concept of abjection which currently remains unused in sociology. Drawing on the example of the fourth age, the article elaborates how analyzing abjection is a crucial part of systematically reconstructing imaginaries. The article concludes that especially the limits of what we can or want to imagine, the exceptional, excluded and barely tolerable are important for understanding the social imaginary.

Keywords Social imaginary $\cdot$ Imagination $\cdot$ Abject $\cdot$ Abjection $\cdot$ Fourth age

Where there is no imagination, there is no horror. (Arthur Conan Doyle)

\section{Einleitung: Leerstellen im Imaginären}

Derzeit ist in der Soziologie eine (wieder-)aufkommende Aufmerksamkeit für Imaginationen und das soziale Imaginäre zu verzeichnen, sei es als empirischer Gegenstand oder als theoretische Kategorie (Gertenbach 2011; Wolf 2012; Adams et al. 2015; Beckert 2018; Delitz 2019; Herbrik und Schlechtriemen 2019). Die Soziologie macht damit eine Lesart von Kultur stark, welche die menschliche Vorstellungskraft als sozial-konstitutive Größe in den Mittelpunkt rückt. Der Begriff des sozialen Imaginären selbst wird dabei in der soziologischen Forschungslandschaft vor allem mit dem griechisch-französischen Philosophen Cornelius Castoriadis in Verbindung gebracht (vgl. z. B. Herbrik und Schlechtriemen 2019), der wesentlich zwischen einem Imaginären (kollektiv getragene Bedeutungsinhalte) und der Imagination (Vorstellungskraft als Vermögen des Individuums) unterscheidet. Mit dieser Unterscheidung trifft die Soziologie des Imaginären - so lässt sich die Bedeutung für das Fach zusammenfassen - eine zentrale Denkfigur der Kultursoziologie: Die Möglichkeit kultureller Hervorbringung (soziales Imaginäres) kann maßgeblich auf die generelle Fähigkeit des Menschen zur Transzendenz (Imagination) zurückgeführt werden (Schütz und Luckmann 2003; vgl. Herbrik 2013). Gesellschaft ist, um mit Castoriadis zu sprechen, eine ,imaginäre Institution“ (Castoriadis 1984) und damit kulturelle Schöpfung (Castoriadis 2010).

Der vorliegende Artikel adressiert ein Desiderat dieser sich gerade institutionalisierenden Soziologie des Imaginären: Während diese vor allem die bedeutungstragenden Inhalte von Imaginationen als Handlungsentwurf, Leitbild oder positivem Gegenhorizont rekonstruiert, bleibt das, was die Grenzen der Vorstellungskraft berührt oder in der individuellen Imagination aversiv erlebt und daher verworfen wird, außerhalb empirischer Forschung wie theoretischer Betrachtungen. Dabei existieren durchaus aktuelle Ansätze in der Kultursoziologie - allerdings ohne expliziten Ver- 
weis auf die soziologische Diskussion zum Imaginären -, die gerade den Vorgang des Ausschlusses und damit auch das Abschreckende, Ausgegrenzte, Abwesende oder Unsagbare selbst als konstitutive Elemente des Sozialen verstehen (Lindemann 2002; Bührmann und Schneider 2008; Giesen 2010; Bröckling et al. 2015a). Diese ,Theorien des Exzeptionellen“ (Bröckling et al. 2015a) markieren gerade nicht die entstandene und empirisch zu beobachtende soziale Ordnung, sondern fokussieren das Inkommensurable und „Andere der Ordnung“ (2015a) als Ausgangspunkt der Rekonstruktion kultureller Phänomene. Im theoretischen Instrumentarium des sozialen Imaginären liegen demgegenüber keine äquivalenten Konzepte für das Unvorstellbare oder Verworfene vor. Doch nur auf dieser Basis würde beschreibbar, wie dasjenige, was (innere oder äußere) Grenzen der Vorstellungskraft berührt, ebenso einen konstitutiven Bestandteil des sozialen Imaginären darstellt. In diesem Verständnis konstituiert sich das soziale Imaginäre aus der Differenz zwischen dem Vorstellbaren und dem Unvorstellbaren: Das Unvorstellbare markiert die konstitutive Grenze alles Imaginären, während das Vorstellbare einen Möglichkeitsraum dimensioniert, der aktualisierte Vorstellungen, aber auch Elemente, die prinzipiell vorstellbar sind, aber momentan (noch) nicht aktualisiert werden, repräsentiert. Das soziale Imaginäre stellt damit zum einen konkrete Vorstellungsinhalte zur Verfügung, die als positive Gegenhorizonte affirmiert werden und als Handlungsentwürfe Umsetzung finden können, zum anderen aber auch aversiv besetzte und abschreckende Inhalte, welche nicht nur als negative Gegenhorizonte vermieden werden wollen, sondern auch radikal verworfen werden.

Es ist der Begriff des Abjekts, wie ihn die französische Philosophin und Psychoanalytikerin Julia Kristeva (1982) konzipiert, der - so das zentrale Argument des vorliegenden Artikels - in besonderer Weise geeignet ist, das letztbenannte Desiderat - das Verworfene im Imaginären - theoretisch zu füllen und damit die Forschung zum sozialen Imaginären zu bereichern, und zwar sowohl für eine weitere theoretische Ausdifferenzierung als auch für empirische Vorhaben.

Begriffe wie Abjekt (abject) oder Abjektion (abjection) sind im deutschen Sprachgebrauch dabei nicht nur alles andere als gebräuchlich, sondern erscheinen als sperrige Neologismen (Menninghaus 2003, S. 366). Am stimmigsten ließe sich „Abjekt“ wohl abgeleitet aus dem Lateinischen ab-iectum tatsächlich als das Ver-worfene übersetzen. Gleichzeitig verweist schon der gleiche Wortstamm von Subjekt, Objekt und Abjekt auf konzeptionelle Bezüge: Abjektes stellt die Grenze von Subjekt und Objekt in Frage und muss daher stets radikal aus dem Bereich dessen, was ein Subjekt ausmacht, ausgeschlossen werden. Kristeva stellt das Abjekt damit als das vor, was den Menschen schmerzlich auf seine Kreatürlichkeit zurückwirft und als Kulturwesen bedroht. Der stete Prozess des Verwerfens, die Abjektion, wird damit zu einer kulturerhaltenden Sicherheitsmaßnahme, da es gerade dieser radikale Ausschluss ist, der dem Subjekt erst ermöglicht, sich zu konstituieren und aufrechtzuerhalten. Mit dem Begriff des Abjekts gelangt die konstitutive Kraft dessen in den Blick, was aus der Vorstellung systematisch ausgeschlossen wird und gerade durch den Prozess des Ausschlusses entscheidende Wirkmacht entfaltet.

Bisher wurden Abjekt und Imagination allerdings nicht in dieser Form systematisch theoretisch zusammengedacht. Paradigmatisch für dieses Desiderat steht die soziologische Auseinandersetzung mit dem sogenannten vierten Alter. Die Sozio- 
logie rekonstruiert das vierte Alter als für die Zukunft gefürchteten imaginierten Zustand. In ihm wird die existenzielle Schwelle von Leben und Tod ausgelotet und markiert, wann ein Körper als Person und wann er als nicht mehr zur Welt des Sozialen gehörend gedeutet wird. Verbunden mit Hinfälligkeit, Demenz und Inkontinenz stellt er ein „Anderes der Ordnung“ dar und widerspricht fundamental allem, was mit moderner Subjektivität verbunden wird. Obschon das Phänomen damit bereits sowohl als soziales Imaginäres als auch als Abjekt gelesen wird (Gilleard und Higgs 2011), bleiben beide Konzepte theoretisch unverbunden. Der vorliegende Artikel führt daher anhand dieses Beispiels vor, wie eine Analyse des Abjekts für die Soziologie des Imaginären nutzbar gemacht werden kann. Hier erweist sich der Begriff, so das Fazit des Artikels, nicht nur als notwendige Binnendifferenzierung des sozialen Imaginären, sondern als gewinnbringende Analysekategorie für eine Soziologie des Exzeptionellen (Bröckling et al. 2015a).

\section{Die Soziologie des Imaginären}

Die Unterrepräsentation des Imaginären im soziologischen Begriffsinstrumentarium ist keinesfalls dessen Irrelevanz für das Fach geschuldet. Ganz im Gegenteil: Seit jeher sind Elemente der Einbildungskraft oder des Entwerfens Teil soziologischer Theorien (Tappenbeck 1999; Herbrik und Schlechtriemen 2019), und bereits bei den soziologischen Klassiker*innen finden sich Verweise auf die Imagination an zentraler Stelle. So sind Handlungsentwürfe (Schütz 1971; vgl. Herbrik und Schlechtriemen 2016), Ideologien und Utopien (Mannheim 1965), Typisierung (Simmel 1992; vgl. Härpfer 2016; Watier 1993) oder auch Prozesse des role-taking (Mead 1973; vgl. Tappenbeck 1999) originäre Leistungen der Vorstellungskraft, die mit jeweils unterschiedlichen Realisierungspotenzialen verbunden sind. Die Fähigkeit zur Perspektivenübernahme, also des imaginierenden Verlassens des eigenen Standpunktes, gilt soziologisch gar als Grundbedingung von Sozialität überhaupt. Ganz konkreten Anschluss in der Soziologie des Imaginären finden zudem Durkheims kollektive und Moscovicis soziale Repräsentationen (Durkheim 1994; Moscovici 2000; vgl. Maffesoli 1993a, 1993b) als konstitutiv begründende Vorstellung einer Gesellschaft von sich selbst.

In den 1990er-Jahren interpretieren Brigitte Fourastié und Philippe Joron (1993) die Hinwendung der Soziologie zum sozialen Imaginären (the imaginary) als eine Anpassung an den sich wandelnden Gegenstand des Faches. Die moderne Gesellschaft sei mehr und mehr von einer ikonographischen Komplexität geprägt, einem „regime of audio-visual communication“ (Fourastié und Joron 1993, S. 55). Inzwischen wird das sozial- und kulturwissenschaftliche Interesse am Imaginären in der internationalen Theorielandschaft als eigenständiges, wenn auch diverses, Feld erkannt (Adams et al. 2015). Während Lacan (2001) den Begriff des Imaginären (vgl. das Symbolische, das Imaginäre und das Reale als Dreigliederung der Psyche) in die akademische Debatte brachte, war es Charles Taylor, durch dessen Modern Social Imaginaries (Taylor 2004) der Begriff der „social imaginaries“ popularisiert wurde (Adams et al. 2015, S. 18f.). Für Taylor handelt es sich beim sozialen Imaginären um eine Art a-theoretisches Wissen im Sinne Mannheims (1964), welches die 
Praktiken der Mitglieder einer Gesellschaft strukturiert (vgl. Binder 2016, S. 537). Taylor stellt das soziale Imaginäre damit ausdrücklich einem (intellektuellen und politischen) Expert*innenwissen entgegen. So stellt es eher ein Repertoire aus implizitem Alltags- und Handlungswissen dar, das sich mehr als in expliziten Theorien in ,images, stories and legends“ dokumentiert (Taylor 2002, S. 106; 2004, S. 23 ). Die politische Soziologie bezieht sich zudem auf Benedict Andersons Imagined Communities (Anderson 1991; vgl. Knaut 2015), welche Nationen als imaginierte Gemeinschaften vorstellt. Eine andere Stoßrichtung schlägt Chris Gilleard (2017) vor. Er liest das soziale Imaginäre nicht als Theorie des Politischen, sondern in einer kultursoziologischen Tradition in einer Linie mit Durkheims und Moscovicis Begriff der Repräsentationen als theoretisches Konzept des ,,how society represents itself to itself“" (Gilleard 2017).

Vor der Popularisierung durch Taylor (2004), arbeitete Cornelius Castoriadis in Gesellschaft als imaginäre Institution (1984; französisches Original 1975) bereits Mitte des letzten Jahrhunderts mit Konzepten des Imaginären. Das Imaginäre versteht Castoriadis als ein komplexes Netz von Bedeutungen (Castoriadis 2010, S. 30), welches jeweils „Aufbau und Gliederung der gesellschaftlichen Welt“ festlegt (Castoriadis 1984, S. 251). „Normen, Werte, Sprache, Werkzeuge, Verfahren und Methoden des Umgangs mit Dingen und ihrer Herstellung, und natürlich das Individuum selbst“" (Castoriadis 2010, S. 29), das alles weist Castoriadis als Institutionen, und damit kulturelle Leistungen (Schöpfung), aus. Mit seiner Frage nach sozialer Ordnung (,was hält eine Gesellschaft zusammen?“) auf der einen und nach sozialem Wandel (,was lässt andere und neue Formen von Gesellschaft entstehen?“) auf der anderen Seite (Castoriadis 2010, S. 27-28) legt Castoriadis im Grunde bereits eine „kulturtheoretisch ausgerichtete Gesellschaftstheorie“ (Gertenbach 2011, S. 278) vor. Schließlich, so betont Heike Delitz (2019), schafft sich jede Gesellschaft auch ein ,zentrale[s] Imaginäre[s]“ (Castoriadis 1984, S. 221), welches ihre kollektive Existenz imaginär in einem Außerhalb ihrer selbst (etwa eines Schöpfergottes) begründet. Das Imaginäre entspricht damit auch der konstitutiven Selbstrepräsentation einer Gesellschaft, indem es die Grundfragen einer jeden Gesellschaft nach deren Identität und Weltdeutung beantwortet (Castoriadis 1984, S. 252).

Im Anschluss an Castoriadis unterscheidet die Soziologie des Imaginären zudem zentral zum einen das Imaginäre als kollektiv getragene Bedeutungs- und Vorstellungsinhalte, zum anderen konkrete Imaginationen in der Vorstellungswelt von Subjekten, welche der Einbildungskraft, dem Vermögen des Individuums zu Fantasie und Vorstellung, entspringen. Die internationale Forschung konzentriert sich, folgt man dieser Unterscheidung, eher auf das soziale Imaginäre als auf individuelle Vorstellungsinhalte und versteht sich als makrosoziologische Kulturanalyse. In den Blick geraten politische und ökonomische Entwicklungen - darunter nicht zuletzt neoliberale Ideologien - sowie Phänomene des sozialen Wandels und sozialer Bewegungen (The Social Imaginaries Editorial Collective 2015, S. 9f.). Insgesamt zeigt das internationale Feld hier einen Schwerpunkt in Themen der politischen Soziologie, welche Gesellschaft als Institution versteht, die sich selbst in je historischen Konstellationen und durch zivilisatorische Aushandlungsprozesse formt (Adams et al. 2015, S. 16). Stärker als die internationale Debatte konzentriert sich die empirisch orientierte deutschsprachige Soziologie auf Imaginationen, also auf 
aktualisierte Bilder und Vorstellungsinhalte - sei es das gemeinsame Schaffen imaginärer Welten beim Rollenspiel (Herbrik 2011, 2013), Zukunftsvorstellungen im Spielfilm (Akremi 2016) oder in Marktvorhersagen (Beckert 2018) - aber auch etwa das Imaginäre der Stadt (Schwenk 2019) oder der Anorexie (Schünzel 2019). Schließlich befragt sie auch die eigene Disziplin nach Vorstellungen des Sozialen und der Gemeinschaft (Niekriez 2011; Schlechtriemen 2014; Meier 2015).

Die Soziologie des Imaginären und der Imaginationen nimmt - so lässt sich die Forschungslandschaft zusammenfassen - vor allem diejenigen Bilder und Vorstellungen in den Blick, die zur Konstitution eines Phänomens oder zur Aufrechterhaltung einer Praxis beitragen, indem sie Imaginationen positiv orientierend füllen. Nichtsdestotrotz stellen Vorstellungsinhalte, welche mit Angst und Schrecken erfüllen und daher ausgeschlossen oder verworfen werden, ebenso einen konstitutiven Bestandteil des sozialen Imaginären dar. Jedoch finden sie in der gegenwärtigen Forschungslandschaft zum sozialen Imaginären keine systematische Umsetzung. Daher verfügt diese auch noch über kein ausdifferenziertes Begriffsinstrumentarium, welches das Verworfene im Imaginären theoretisch $\mathrm{zu}$ benennen und konzeptionell $\mathrm{zu}$ fassen vermag.

\section{Notwendige Ergänzungen: Abjekt und Abjektion}

Kristevas Konzept des Abjekts eignet sich aus mehreren Gründen dafür, diese theoretische Leerstelle zu füllen. Zum einen beschreibt sie mit Abjektion genau jenen Vorgang der Verwerfung, der in der Soziologie des Imaginären bisher unbestimmt bleibt. Zum anderen verorten sowohl Kristeva als auch Castoriadis, der zentrale Referenzautor der Soziologie des Imaginären, die Grundlegung ihrer Theorie in der Psychoanalyse und teilen daher wesentliche anthropologische und sozialisationstheoretische Prämissen, indem sie die Möglichkeit kultureller Schöpfung mit der Genese und Konstitution des Subjekts verknüpfen. Schließlich lässt sich über das in Castoriadis' Kulturverständnis zentrale Konzept des „Magmas“ der Schlüssel zur Anknüpfung der Soziologie des Imaginären an die Denkfigur der Abjektion als Kulturschöpfung herleiten.

Während die Begriffe des Abjekts und der Abjektion in die Kunst- und Kulturtheorie bereits Eingang gefunden haben (Schmitz 2000, S. 37; Menninghaus 2003, S. 365), sind sie in der Soziologie bisher nur am Rande beachtet worden. Dass z. B. Judith Butler die Begriffe abject und abjection nutzt, um die Wirkmacht der heterosexuellen Matrix (Butler 1997) zu veranschaulichen, ist durch die Übersetzung ins Deutsche weitgehend verloren gegangen. ${ }^{1}$ Butler stellt der Möglichkeit des Subjekts, sich mit einem Geschlecht zu identifizieren, die Bedingung voran, dass diskursiv bestimmte Identifizierungen angeboten und andere aus dem Möglichkeitsraum verworfen werden. Das Verworfene bezeichnet bei ihr entsprechend

\footnotetext{
1 Lediglich bei der ersten Nennung des „Verworfenen“ auf S. 23 in Körper von Gewicht (Butler 1997) wird das Original abject in Klammern genannt und in der zugehörigen Fußnote kurz erläutert. In der weiteren Übersetzung werden die Begriffe ,das Verworfene“ und die teilweise etwas sperrigen Übersetzungen für abjection „Verworfenheit“, „Verwerflichkeit“ und „Verwerflichmachung“ genutzt.
} 
die ,,nicht lebbaren“ und ,unbewohnbaren“ Zonen des sozialen Lebens“, welche sie mit dem ,konstitutive[n] Außen zum Bereich des Subjekts“ assoziiert (Butler 1997, S. 23). Martina Biebert und Michael Schetsche (2016) wiederum entlehnen den Begriff des Abjekts aus dem psychoanalytischen Zusammenhang und übertragen ihn in metaphorischer Weise auf die Makroebene. Unter dem von ihnen gebildeten Konzept der „kulturellen Abjekte“ verhandeln sie „dauerhaft unintegrierbares Wissen“ einer Gesellschaft oder Kultur. Sie nutzen den Begriff also vor allem als Analogie und adressieren eine Form ausgeschlossener gesellschaftlicher Wissensbestände (z. B. Homosexualität im Fußball). Schließlich beziehen sich Chris Gilleard und Paul Higgs in ihrem Artikel Aging Abjection (2011) sowohl auf den Begriff des Abjekts als auch auf den des sozialen Imaginären, indem sie das vierte Alter als soziales Imaginäres verstehen, das vor allem durch den ,horror of abjection“ (Gilleard und Higgs 2011, S. 137) geformt wird. Allerdings buchstabieren sie die Relation der Konzepte des Imaginären und des Abjekts auf einer theoretischen Ebene nicht aus.

Kristeva selbst entfaltet ihre Ausführungen, indem sie Konzepte der Psychoanalyse (1982, S. 32ff.) mit der zeitgenössischen Anthropologie (1982, S. 56ff.) verbindet. Für Kristeva ist das Abjekt das Verworfene, das radikal aus dem Bereich dessen, was ein Subjekt ausmacht, Ausgeschlossene. Sie stellt es als das vor, was den Menschen mit seiner auch schwer kontrollierbaren Körperlichkeit konfrontiert (z. B. Regelblutung, Ausscheidungen) und was als Unreines mit Gefühlen von Angst und Scham - aufgrund des Scheiterns des Managens der Körpergrenzen - verbunden ist. Es ist etwas, auf das wir nachdrücklich aversiv reagieren und das daher im Alltag vehement ausgeschlossen werden muss. Doch, und das ist der Kunstgriff Kristevas, ist es gerade dieser Ausschluss, der es dem Subjekt ermöglicht, sich selbst zu konstituieren und als Subjekt aufrechtzuerhalten. Denn das Abjekt, so argumentiert sie in der Tradition der Psychoanalyse, ist kein bloßes Objekt, das dem Subjekt schon immer gegenübersteht, sondern verweist stets auf das Subjekt und die Grenzen, die durch Verwerfungen gezogen worden sind und die das Abjekt markieren (Kristeva 1982, S. 6). So verortet sie in Auseinandersetzung mit Freud und Lacan den Ursprung jeglicher Form von Subjektivität im Prinzip einer frühkindlichen, noch in einer präoralen Phase stattfindenden, Trennung von Subjekt und Objekt (Kristeva 1982, S. 14; vgl. Hövelmeyer 2007, S. 91). Auch Castoriadis setzt sich mit der Psychoanalyse auseinander (z. B. 1984, S. 497-509, 2012). Allerdings revidiert und erweitert er diese. So sind etwa Freuds Konzeptionen des Unbewussten und der Fantasie für Castoriadis zu kurz gefasst. Denn Freud spricht von der Fantasie immer nur als einem kompensatorischen Akt, d.h. die Fantasie wird erst dann aktiv und Vorstellungen werden erst dann erzeugt, wenn ein Bedürfnis nicht befriedigt ist, dem Subjekt also etwas fehlt und ein Mangel vorhanden ist (Castoriadis 2012). Demgegenüber macht Castoriadis Imagination nicht im Rekurs auf andere Funktionen verständlich, sondern gründet sie in dem, was er als „Magma“ bezeichnet, einer „Schicht des Vorfindlichen“, das den undifferenzierten Urgrund für jedwede ontologische Schöpfung (Castoriadis 1984, S. 384) und damit die Grundlage für alles Imaginäre bietet. Mit der Metapher des „Magmas“, welche Fluidität kennzeichnet, ohne dabei Beliebigkeit zu implizieren, bezeichnet Castoriadis die ,Seinsart des Gegebenen [...], bevor es von der Identitäts- und Mengenlogik geprägt worden ist" (Castoriadis 1984, S. 564), also bevor Bedeutungen festgeschrieben werden. Das Magma als Möglichkeitsuniversum 
umfasst damit immer auch dasjenige, das bei der Konstitution von Bedeutung verworfen wurde. Es beinhaltet auch diejenigen Bedeutungsmöglichkeiten, die nicht verwirklicht worden sind und sich damit einer Festschreibung und Benennung (für den Moment) entziehen. Diese verworfenen Möglichkeiten bezeichnet Castoriadis als „Rauschen“ (Castoriadis 2010, S. 32), welches damit das begriffliche Äquivalent zum Abjekt darstellt.

Castoriadis sieht das Magma des Unbewussten als Grundlage, aus der sowohl das Subjekt und sein Bewusstsein, als auch gesellschaftliche Ordnung geschöpft werden. Wie Freud geht er davon aus, dass sich das Subjekt aus einer psychischen Monade entwickelt, für welche zunächst keine Trennung von Ich und Welt erlebt wird. Diese Trennung wird für Castoriadis durch die Umwelt vollzogen, Sozialisation ist also mit der Öffnung der psychischen Monade verbunden. Für die Monade stellt diese Trennung, die Unterbrechung des unaufhörlichen Vorstellungsflusses, entsprechend einen Bruch mit sich selbst dar (Castoriadis 1984, S. 499).

Für Kristeva hingegen vollzieht sich die Subjektwerdung durch einen von der psychischen Monade selbst initiierten Ausschluss: Damit überhaupt eine Grenze zwischen Körper und Umwelt gezogen werden kann, muss, so Kristeva, etwas aus dem undifferenzierten Urgrund abgegrenzt, abgesondert, also als Teil des Körpers verworfen werden. Zwischen dem Körper und dem Verworfenen wird damit ein aversives Verhältnis gesetzt. Dieser so als Anderes objektivierte Selbstanteil wird als Abjekt bezeichnet. Damit wird der Prozess des Verwerfens zur konstitutiven Grundbedingung des Subjekts. Gleichzeitig handelt es sich bei der Abjektion nicht um einen einmaligen Vorgang, sondern um einen steten Prozess, der beständig vollzogen werden muss. Denn das Abjekt wird durch den Ausschluss nicht einfach annihiliert, sondern bleibt bedrohlich (Kristeva 1982, S. 6), indem es die Grenze zwischen Subjekt und Objekt zu verwischen droht und damit den Menschen in seinem Sein als Kulturwesen elementar in Frage stellt (Kristeva 1982, S. 4). Das Subjekt muss sich vor dem Abjekt, das zwar eigentlich Teil des Selbst, aber zugleich nicht tolerierbar ist (z.B. der Tod oder die Exkremente), schützen und dieses daher radikal ausschließen, also verwerfen (Kristeva 1982, S. 2). Dabei kommen alle Strategien des Ausschlusses ,niemals an ein Ende, weil sie in ihren Versuchen, das Andere der Ordnung zum Verschwinden zu bringen, diese stets neu hervorbringen“" (Bröckling et al. 2015b, S. 13).

Gleichzeitig beschreibt Kristeva mit dem Lacan'schen Begriff der ,Jouissance“ eine eigentümliche Faszination, eine Ekel-Lust, die vom Abjekt ausgeht (1982, S. 9f.). So erliegt der Surrealist Bataille (1972) den powers of horror, indem er eine wahre Passion für das Abjekt entwickelt. In der Hingabe an das Obszöne, die Ekstase und die Verausgabung hat sich sein literarisches Werk ganz einem genüsslichen Auskosten von Abjektem verschrieben. Und unter dem Label der abject art firmiert sogar eine ganze Kunstform, die mit der dem Abjekt innewohnenden Gleichzeitigkeit des Abscheus und der Faszination, des Abwertens und des Auskostens spielt - und nicht selten provoziert.

Abjection und abject - zweites sowohl als Adjektiv wie als Substantiv lesbar (vgl. Menninghaus 2003, S. 365) - werden, so lässt sich zusammenfassend festhalten, in zweifacher Weise verwendet: Zum einen wird mit dem Begriff Abjektion der Prozess des Ausschließens bezeichnet - in der frühkindlichen Subjektkonstitution 
ebenso wie in der späteren Aufrechterhaltung des Subjektstatus. Das Abjekt ist dabei das Verworfene, Ausgegrenzte. Zum anderen bezeichnet Abjektion aber auch den leiblich-emotionalen Zustand des Subjekts, welches sich mit Abjektem konfrontiert sieht. Abjekt ist dabei all jenes, welches diesen Zustand auslöst. Damit ist es immer nur in Relation zum Subjekt als dessen zu verwerfender Selbstanteil bestimmbar.

\section{Abjekt und Imagination: Das Verworfene im Imaginären des vierten Alters}

Die soziologische Forschung zum sogenannten vierten Alter steht paradigmatisch für die notwendige Ergänzung des Theorieinstrumentariums des sozialen Imaginären um den Begriff des Abjekts. So gibt es zwar einzelne Ansätze, das vierte Alter als soziales Imaginäres und als Abjekt zu fassen, jedoch bleiben beide Konzepte auf theoretisch-konzeptioneller Ebene stets unverbunden. Demgegenüber möchte ich im Folgenden an diesem Beispiel zeigen, wie das Abjekt systematisch als Element des Imaginären konzipiert werden kann. Zunächst wird dargestellt, wie das vierte Alter als soziales Imaginäres begriffen werden kann. Die Analyse als Abjekt erfolgt daraufhin in drei Schritten: Erstens wird analysiert, welche existenziellen Grenzüberschreitungen durch das vierte Alter repräsentiert werden; zweitens, wie diese Grenzüberschreitungen kulturell eingehegt werden; und drittens, wie sich die moralische Ordnung des vierten Alters in Formen des sozialen Ausschlusses verwirklicht. Schließlich werden Rückschlüsse für eine soziologische Analyse des Abjekts gezogen und dargestellt, welcher Mehrwert sich durch die Zusammenführung der Konzepte Abjekt und Imagination ergibt.

\subsection{Das vierte Alter als soziales Imaginäres: ein ,state of becoming“}

Gilleard und Higgs sind die zentralen Autoren, die das vierte Alter als soziales Imaginäres vorstellen. Für ihre Ausführungen greifen sie die Unterscheidung von drittem und viertem Alter auf, wie sie von Peter Laslett (1989) als Kategorien zur Einteilung der späten Lebensphase eingeführt wurden. Anstatt drittes und viertes Alter allerdings als Komplementärkategorien zur Beschreibung der empirischen Realität von aufeinanderfolgenden, wenn auch radikal unterschiedlichen, Phasen zu konzipieren, sehen Gilleard und Higgs in ihnen ,different paradigms“ (Gilleard und Higgs 2013, S. 368), mit denen Alter grundlegend anders konzipiert wird. Das dritte Alter kann als ein Feld begriffen werden, das durch einen spezifischen lifestyle der jungen oder aktiven Alten (vgl. van Dyk und Lessenich 2009) repräsentiert und gelebt wird. Reproduziert wird es durch mit diesem lifestyle verbundene alltägliche Praktiken des Freizeitverhaltens, des Konsums und der Selbstverwirklichung, aber auch durch ehrenamtliche Tätigkeiten (Gilleard und Higgs 2013, S. 369). Im Gegensatz dazu kann das vierte Alter als soziales Imaginäres verstanden werden, denn es speist sich vor allem aus Vorstellungen, die zudem nicht einer eigenerlebten Erfahrung entspringen. Repräsentiert und reproduziert wird es durch kulturelle Narrative und Vorstellungswelten, die von Hinfälligkeit und sozialem Ausschluss der Betroffenen geprägt sind (Gilleard und Higgs 2013, S. 368). Wenn ältere Menschen im öffentli- 
chen Diskurs adressiert werden, dann als third agers und damit verbunden als aktive und selbstsorgende Subjekte. Das vierte Alter hingegen bietet keine Identifikationsfläche für das Hier und Jetzt, sondern repräsentiert einen für die Zukunft prospektiv gefürchteten „,state of becoming', an ascribed community of otherness, set apart from the everyday experiences and practices of later life" (Gilleard und Higgs 2013, S. 368) $)^{2}$.

Vermittelt werden diese Vorstellungen im kulturellen Repertoire der modernen Gesellschaft als social imaginary über Bilder, Geschichten und Erzählungen (Taylor 2004). Gerade in den letzten Jahren sind international und auch in deutscher Sprache zahlreiche Romane und Spielfilme erschienen, die sich mit dem Thema Demenz befassen (Zeilig 2014), über welches das vierte Alter vor allem adressiert und ,popularisiert“ (Swinnen und Schweda 2015) wird. Mit der zunehmenden Konfrontation mit dem vierten Alter in langlebigen Gesellschaften findet sich auch ein gesteigertes Interesse an Demenz-Erzählungen (Völk 2019, S. 365 und 371). Der Schrecken des sozialen Imaginären des vierten Alters dokumentiert sich schließlich auch in alarmistischen und apokalyptischen demographischen Szenarien der „alternden Gesellschaft", wie sie nicht nur in den Massenmedien (Katz 1992; Gee und Gutman 2000; vgl. Katz und Whitehouse 2017), sondern auch in Form von Expert*innenwissen durch die biomedizinische Demenzforschung (Schicktanz 2017) präsentiert werden.

\subsection{Grenzüberschreitungen: von Zombies und Schläuchen}

Gilleard und Higgs beschreiben das vierte Alter zudem auch explizit mit den Begriffen des Abjekts und der Abjektion, denn es ist verbunden mit einem ,horror with which ,real" old age is viewed“" (Gilleard und Higgs 2011, S. 138). Dieser horror lässt sich mit Kristeva in erster Linie aus dem Potenzial des Abjekten zur Grenzüberschreitung erklären:

It is thus not lack of cleanliness or health that causes abjection but what disturbs identity, system, order. What does not respect borders, positions, rules. The inbetween, the ambiguous, the composite (1982, S. 4).

So sind Dinge nicht allein deshalb abjekt, weil sie beispielsweise schmutzig sind oder Krankheiten auslösen können, sondern weil die Vermischung getrennt zu haltender Sphären als unrein erlebt wird. Nach Kristeva überschreitet das Abjekt die Grenze zwischen Subjekt und Objekt (Kristeva 1982, S. 4), zwischen Innen und Außen des Selbst (1982, S. 53), zwischen Innen und Außen des Körpers (1982, S. 101), zwischen Natur und Kultur (1982, S. 75) und zwischen Menschlichem und NichtMenschlichem (1982, S. 75). Die Repräsentationen dieser Grenzüberschreitungen im sozialen Imaginären des vierten Alters zeigen eindrücklich die Interviewstudien von

\footnotetext{
2 Was Gilleard und Higgs nicht erwähnen, ist, dass prinzipiell auch ein Feld des vierten Alters (Biographien des langen Lebens, Pflege von Angehörigen, Pflegeberufe) existiert. Umgekehrt stellt das dritte Alter auch ein soziales Imaginäres dar, das im Gegensatz zum vierten Alter allerdings als positive Projektionsfläche (späte Freiheit, Reisen, Selbstverwirklichung) fungiert. Empirisch lässt sich ein gelebter Alltag allerdings vom sozialen Imaginären als (noch) nicht-gelebte (idealisierte oder gefürchtete) Vorstellung der (eigenen) Zukunft klar unterscheiden.
} 
Els van Wijngaarden et al. (2017). In der das vierte Alter begleitenden Metaphorik werden die (zukünftigen) Körper der Betroffenen sogar von diesen selbst durch den Vergleich mit Müll (,,piece of dirt“) oder ausrangierten Maschinen (,wreck“) in die Sphäre der Materie, aber auch als Schlachtvieh (,slice of meat") und vertrocknende Pflanzen (,shriveled“) in die Sphäre des Kreatürlichen verwiesen (van Wijngaarden et al. 2017). Die Überschreitung der Grenze zum Nicht-Menschlichen und Monströsen (Bülow und Holm 2016 mit Verweis auf Shildrick 2001) im vierten Alter wird auch im Vergleich von Demenzkranken mit Zombies (,animated corpse“) deutlich (Behuniak 2011).

In der Sprache einer Soziologie des Exzeptionellen (Bröckling et al. 2015a) erscheint das soziale Imaginäre des vierten Alters als Beispiel eines „Grenzregimes“ (Lindemann 2011). Es symbolisiert den Verlust der eigenen Identität und Selbstwirksamkeit und kann durch die Einbuße von ,,social agency“ (Gilleard und Higgs 2011, S. 138) mit einem sozialen Tod gleichgesetzt werden (Gilleard und Higgs 2015). Damit wird das vierte Alter in den Bereich des kulturell Unverfügbaren verwiesen. Es ist ein Zustand, in welchem man - so Teilnehmer*innen in einer Gruppendiskussion zum Thema Patient*innenverfügung - als ,ein lebend Stück Fleisch“ nur noch „gefüttert werden“ kann (Brauer et al. 2014, S. 440). Im Gegensatz dazu wird bei kleinen Kindern das Gefüttert-Werden nicht in dieser Art und Weise problematisiert. Zwar verfolgt beides den gleichen Zweck, jedoch steht das Füttern im Alter stets unter dem Vorzeichen des Verlustes von Kompetenz und Selbstständigkeit. Das in der Pflege verwendete wording vom „Angeben“ des Essens verweist noch auf die einst bestehende Fähigkeit, selbstständig Nahrung aufzunehmen. Der Zustand des Gefüttert-Werdens, der bei Babys mit Hinwendung, Liebe und Sorge gesehen wird, muss am Lebensende als Ausdruck von Abhängigkeit radikal - und letztlich auch mit aller Härte gegen sich selbst - abgewendet werden. Es genügt eine indexikale Andeutung (z. B. „Schläuche“), um das Schreckenspotenzial des Imaginären des Lebensendes unter den Voraussetzungen der Apparatemedizin aufzurufen (Brauer et al. 2014, S. 436). Das Eingreifen der Apparate in den menschlichen Körper und damit die Behandlung dessen als bloße Materie stellt eine für die fundamentale Trennung von Subjekt und Objekt nicht tolerierbare Vermischung des Organischen mit Anorganischem, des Menschlichen mit Nicht-Menschlichem, des Innen und des Außen dar.

Mit dem Begriff des Abjekts lässt sich nun die Vehemenz und Radikalität dieser Abwehrhaltung gegenüber Vorstellungen des vierten Alters auch theoretisch fassbar machen. Denn zur Aufrechterhaltung des Subjektstatus ist eine radikale Verwerfung (und nicht etwa nur Erduldung oder Bearbeitung) all dessen notwendig, was mit Vorstellungen moderner Subjektivität nicht vereinbar scheint. Besonders betrifft dies die Vorstellung von Demenz und Inkontinenz (Bielefeldt 2016). So wird in literarischen Bearbeitungen des vierten Alters (wie etwa in David Wagners „Der vergessliche Riese"), aber auch in populärwissenschaftlichen Beiträgen (z.B. die Spiegel-Wissen-Ausgabe „Reise ins Vergessen. Leben mit Demenz“) im Besonderen das Vergessen der eigenen Biographie, aber auch von nahestehenden Personen thematisiert. Im Film „Still Alice“ bricht die Protagonistin zum ersten Mal in verzweifeltes Schluchzen aus, als sie von ihrer Angst berichtet, durch ihr Vergessen ihr Leben zu verlieren, und zum zweiten Mal, als sie nicht verhindern kann, sich 
in die Hose zu pinkeln. Auch hier lässt sich die Vergleichsfolie der frühen Kindheit heranziehen, denn der Ekel vor den Exkrementen des (eigenen) Kindes wird kulturell ganz anders bearbeitet als mit einer solch existenziellen und sprachlosen Verzweiflung mit Blick auf das vierte Alter.

\subsection{Einhegepraktiken: bürokratische Sicherungsverfahren, ,,radikale Fiktion“ und Idealisierung}

Mit dem Zusammendenken der Kategorien der Imagination und des Abjekts gelingt es nicht nur, das Verworfene selbst zu charakterisieren und die radikale Abwehrhaltung gegenüber dem sozialen Imaginären des Lebensendes aus dem transgressiven Potenzial des Verworfenen erklärbar zu machen. Durch die psychoanalytische Herleitung des Mechanismus der Abjektion durch Kristeva kann auch die konstitutive Verwiesenheit des Abjekts auf dasjenige, was es zu schützen gilt, benannt werden: Der Status der Person. Personale agency (Emirbayer und Mische 1998) zeichnet sich durch Selbstbestimmung und die Fähigkeit aus, Entscheidungen zu treffen. So existieren auch für das Lebensende moderne bürokratische Sicherungsverfahren als prospektive Verwaltung der Grenzen des eigenen Körpers und der Person, wie etwa die Patient*innenverfügung (Brauer et al. 2014), welche die medizinisch begleitete Phase vor dem Tod zu regeln versucht. Solche Verfahren lassen sich mit Bernhard Giesen als Praktiken des Einhegens beschreiben, die sich ,zwischen uns und den monströsen Rohzustand der Welt" schieben (Giesen 2010, S. 59ff.). Diese Einhegepraktiken werden notwendig, da dasjenige, was kulturelle Grenzen überschreitet, also die soziale Ordnung missachtet, nicht nur mit Gefahr assoziiert ist, sondern mit Angst und Schrecken erfüllt. Das Ausfüllen des Dokuments bearbeitet diese Grenzen, indem es vermeintliche Eindeutigkeiten festlegt, damit bedrohende mögliche Ambivalenzen und Indifferenzen (Giesen 2010) überschreibt, um abjekte Imaginationen ,ad acta“ zu legen. Letztlich allerdings wendet es damit den sozialen Tod durch das letzte Mittel des körperlichen Todes ab.

Brauer et al. (2014) deuten die Entscheidung gegen das eigene Leben als „radikale Fiktion“ der letzten Möglichkeit, den Status als soziale Person prospektiv aufrechtzuerhalten und damit Handlungssicherheit im Hier und Jetzt herzustellen. Der radikale Ausschluss abjekter Vorstellungsinhalte - bis hin zur Forderung des eigenen Todes - wird damit zum konstitutiven biographischen Element. Eine ganz andere Einhegepraktik stellt die der Idealisierung der Demenz dar. Dementsprechend wird in der Populärkultur die Demenz auch als Zustand einer neuen, vielleicht vorher nie geahnten, emotionalen Tiefe dargestellt. So beschreibt etwa Arno Geiger in „Der alte König in seinem Exil““, wie durch die Erkrankung seines Vaters ihre Beziehung nicht nur durch eine neue emotionale Ebene bereichert, sondern überhaupt gar erst ermöglicht wurde. Damit stellen Idealisierungen und Glorifizierungen neue Vorstellungen zur Verfügung, die den Schrecken des vierten Alters auszublenden verhelfen.

In diesen Einhegepraktiken findet sich verwirklicht, was Bröckling et al. als kulturelle Bearbeitung des „Anderen der Ordnung“ verstehen. Denn dieses muss stets ,beseitigt, überwunden, normalisiert werden. Dies kann durch Bekämpfung und Ausstoßung erfolgen, aber auch durch Neutralisierung seiner Alterität“" (2015b, 
S. 13). Während die „,radikale Fiktion“, die reflexhafte Entscheidung für den eigenen Tod, eine Form der Ausstoßung und die Bürokratie eine Form der Normalisierung darstellt, kann die Idealisierung des vierten Alters als eine Art Neutralisierung verstanden werden.

\subsection{Moralische Ordnung: Das Othering des vierten Alters}

Abjektion spielt sich nicht nur auf Ebene des Individuums ab, sondern entfaltet kulturell eine normative Dimension. Georges Bataille führt in seinem Aufsatz Abjection and Miserable Forms (1999; vgl. Gilleard und Higgs 2011) aus dem Jahr 1930 den sozialen Ausschluss und die kulturelle Abwertung der unteren Schichten darauf zurück, dass diese durch die harte Arbeit, die sie verrichten, und die elenden Zustände, in denen sie leben müssen, strukturell nicht in der Lage sind, Schmutz und Krankheit von sich fernzuhalten. Und auch die Überlegungen Kristevas bleiben nicht auf innerpsychische Vorgänge beschränkt, auch sie denkt Abjektion nicht außerhalb von Machtverhältnissen (vgl. Gilleard und Higgs 2011, S. 136). Während Bataille die Kategorie der Klasse in den Blick nimmt, ist es bei Kristeva das Geschlecht, welches sie als Machtverhältnis denkt. Denn es sind vor allem die weiblichen Körper, deren Körperflüssigkeiten (Menstruationsblut) als unrein gelten, und die als Mütter (und Pflegende) auch mit Exkrementen umgehen müssen. Die „maternal authority“ wird zum Garanten des Reinen und unterscheidet in einer binären Logik zwischen ,proper-clean and improper-dirty“ (Kristeva 1982, S. 71f.). Die beständige Arbeit an der Grenze von Rein und Unrein wird in ihrer Denkfigur als die elementare Grundlage von Kultur dargestellt: ,There, abject and abjection are my safeguards. The primers of my culture“ (Kristeva 1982, S. 2). So gelingt es Kristeva, über die Verbindung von Psychoanalyse und Anthropologie (Mary Douglas) die psychische Konstitution von Subjekten mit der Morphologie des Sozialen zu verbinden (1982, S. 69).

$\mathrm{Zu}$ befragen wären also schließlich die normativen Gehalte der Abjektion im sozialen Imaginären des vierten Alters und hiermit die Folgen für die Betroffenen (Demente/Pflegebedürftige). Durch das Othering des vierten Alters (Higgs und Gilleard 2014; van Dyk 2016) wird nicht nur Abjektes systematisch aus der Vorstellung und damit aus der Kultur verdrängt; es bedeutet gleichzeitig einen „social death“ (Gilleard und Higgs 2015) der Betroffenen, also ihren Ausschluss aus der ,moral community“ (Bataille 1999, S. 10). So ist das Verwerfen verbunden mit einer Dehumanisierung (,vegetieren“, ,,abschalten“) und einer Aberkennung des Status als Person. Die „Barbarisierung des Alters“ (Gronemeyer 2004) berührt so letztendlich Fragen der Menschenwürde (Bielefeldt 2016). Aber nicht nur Dehumanisierung, sondern auch die beschriebene Einhegepraktik der Idealisierung ist eine Form des Othering: So sind das emotionale „Aufblühen“ der Demenzkranken oder eine neue Qualität und Tiefe von Beziehungen, wie sie in fiktionalen Werken zuweilen beschrieben werden, durchaus auch als romantisch verklärte „Pathosformeln“ (Völk 2019, S. 368ff.) zu lesen.

Letztlich bleibt der „Ordnungsbias“ (Bröckling et al. 2015b), der sich in der asymmetrischen Differenzkategorie des Alters (van Dyk 2016) verwirklicht, auch für die wissenschaftliche Beschäftigung nicht ohne Folgen. Die anhaltende gerontologische Rede vom Successful Aging legt den Fokus auf das aktive und produktive Altern, 
schließt damit andere Inhalte aus (Schroeter 2004; Pfaller und Schweda 2017) und erklärt sie zum „Rest“, um eine der Metaphern zur Bestimmung des Inkommensurablen (Bröckling et al. 2015b, S. 41) zu gebrauchen. Higgs und Gilleard verbinden das Konzept des vierten Alters daher mit der Metapher des „black hole“ (Gilleard und Higgs 2010, S. 121), welches sich jedweder Annäherung entzieht und aus dem nichts nach außen dringen kann: „The loss of agency, of identity, casts those with neither voice nor choice into a position of exclusion and marginality - a kind of dark matter whose presence is felt but never identified" (Higgs und Gilleard 2017, S. 66).

\subsection{Zusammenfassung: Subjekt und soziale Ordnung in der Abjektanalyse}

Durch das Zusammenführen der Konzepte des Abjekts und des Imaginären lässt sich das Phänomen des vierten Alters zwischen den Analysekategorien soziales Imaginäres, Imagination, Abjekt und Abjektion aufspannen: Das soziale Imaginäre des vierten Alters zeigt sich als in die Zukunft imaginierter körperlicher und sozialer Status. Der im sozialen Imaginären des vierten Alters eröffnete Möglichkeitsraum bietet allerdings kaum positive Vorstellungsinhalte, also konkrete Imaginationen, an, auf die Subjekte in Form von Handlungsentwürfen affirmativ Bezug nehmen könnten - beispielsweise in Form einer liebevoll zugewandten Pflege und eines UmsorgtWerdens. Vielmehr ist das Imaginäre des vierten Alters von Vorstellungsinhalten geprägt, die schmerzlich auf die eigene Verletzlichkeit zurückwerfen. Die auf Ebene der individuellen Vorstellung aktualisierten Szenarien sind damit nicht nur ein negativer Gegenhorizont, der mit einem positiven abzuwägen wäre. Als Abjekt, das den eigenen Subjektstatus bedroht, können sie nur verworfen werden. Doch werden nicht nur aus der Gefühlswelt der Individuen abjekte Vorstellungen ausgeschlossen, auf sozialer und institutioneller Ebene hat die Abjektion die Exklusion der Betroffenen zur Folge. Denn das Abjekt bedroht das Subjekt und die soziale Ordnung gleichermaßen, da allein schon die Möglichkeit kultureller Schöpfung mit der Genese und Konstitution des Subjekts verknüpft ist. Der methodische Mehrwert einer soziologischen Analyse des Abjekts zeigt sich dann auch darin, dass sie neben der affektiven auch die normative Dimension der Abjektion auslotet und damit nicht nur auf der Ebene des Individuums verbleibt, sondern gerade auch die kulturelle Ebene vermisst. So spiegelt sich in der Abjektion immer auch eine moralische Ordnung wider. Damit schärft ein Einbezug des Begriffs des Abjekts nicht nur das Verständnis eines spezifischen Phänomens, sondern auch das soziale Imaginäre als theoretische Kategorie.

\section{Fazit: Das Imaginäre und seine Grenzen}

Man muss nicht so weit gehen, das Imaginäre zu einer ,theoretischen Zentralkategorie“ (Gertenbach 2011, S. 285) der Soziologie erheben zu wollen. Allerdings werden mit dem wechselseitigen Bezug von Imagination und Imaginärem in der Tat zentrale Fragen nach dem Entstehen, dem Aufrechterhalten und der Veränderung sozialer Ordnung sowie dem Verhältnis von Individuum und Gesellschaft adressiert. 
Nicht nur öffnet oder beschränkt soziales Imaginäres den Möglichkeitsraum der individuellen Imagination, umgekehrt ist es selbst grundlegend an die Möglichkeit des Individuums gebunden, bestimmte Vorstellungsinhalte zu aktualisieren. Gerade die Frage nach dem Verworfenen ist es allerdings, die bisher in den Forschungsvorhaben der Soziologie des Imaginären noch nicht umfassend umgesetzt wurde. Dabei beraubt sich die Analyse entscheidender Aspekte, wenn Vorstellungen stets nur als positiv orientierende Leitbilder oder umzusetzende Handlungsentwürfe verstanden werden und die konstitutive Kraft des Verworfenen unbeachtet bleibt.

In diesem Zusammenhang stellt der Begriff des Abjekts eine bisher für die Soziologie noch nicht ausbuchstabierte, jedoch theoretisch interessante Kategorie dar, eröffnet es doch den Blick für die Funktionsweise des sozialen Imaginären, das immer auch durch das in ihm Verworfene konstituiert wird. Am Beispiel des vierten Alters konnte entsprechend vorgeführt werden, wie der Imagination stets die Abjektion zur Seite steht. Die grundlegende Ordnung des sozialen Imaginären lässt sich damit nicht nur als durch äußere Grenzen (das prinzipiell Unvorstellbare) dimensioniert erschließen. Für das vierte Alter ließen sich vor allem unterschiedliche Manifestationen des Abjekts als unterschiedliche existenzielle Grenzüberschreitungen erkennen und damit Rückschlüsse auf Grenzziehungen innerhalb des sozialen Imaginären des vierten Alters ziehen. Schließlich konnten soziale Praktiken rund um das vierte Alter (bspw. Patient*innenverfügungen) als kulturelle Bearbeitungen des Abjekts konzeptionell erfasst werden.

Natürlich stellt das soziale Imaginäre des vierten Alters nur ein Beispiel dar. Doch ist das Abjekt als Teil des Imaginären komplementär zu affirmativen Vorstellungsinhalten und Leitbildern - gleichsam als „,dunkle Seite der Imagination“ - immer mitzudenken und in die Theorie des sozialen Imaginären zu integrieren. Dabei bedeutet Abjektion nicht, dass abjekte Vorstellungsinhalte keine Handlungsrelevanz entfalten. Ganz im Gegenteil ruft die Konfrontation mit dem Abjekt, wie das Beispiel des vierten Alters eindrücklich zeigt, nicht nur eine Reihe von kulturellen Einhegungen auf den Plan, sondern führt zur Exklusion der Betroffenen. Fraglos sind soziale Ungleichheit und symbolische Grenzziehungen, seien sie geschlechts(Bourdieu 2005) oder schichtspezifisch (Lamont 1992), Kernfragen der Soziologie. Eine Theorie der Abjektion eröffnet hierbei allerdings einmal mehr den Blick für die affektive Ordnung symbolischer Grenzen und Machtstrukturen.

Hier zeigt sich der Mehrwert einer soziologischen Abjektanalyse, die nicht auf Ebene des Subjekts verbleibt, sondern die soziale Dimension des Ausschlusses als Othering mitdenkt. So trägt der Begriff des Abjekts nicht nur zu einer Binnendifferenzierung des Konzepts des sozialen Imaginären bei. Auch steht einer ,Soziologie jenseits des Ordnungsbias“, so die Autor*innen des Forschungsprogramms der Soziologie des Exzeptionellen, bisher ein weit weniger ausgearbeitetes Instrumentarium zur Beschreibung ihrer Phänomene zur Verfügung als einer Soziologie, die auf Ordnungsphänomene fokussiert und hier auf etablierte Begrifflichkeiten und theoretische Differenzierungen zurückgreifen kann (Bröckling et al. 2018, S. 258). Mit der Einführung des Begriffs des Abjekts wird damit das Vorhaben, das ,Soziale von seinen Grenzen her" (Lindemann 2009) zu denken, nicht nur für die Soziologie des Imaginären begrifflich entscheidend vorangebracht. 
Danksagung Ganz besonders bedanken möchte ich mich bei den anonymen Gutachter*innen, die sich die Zeit genommen haben, diesen Artikel mit ihren wertvollen Hinweisen und hilfreichen Kommentaren zu unterstützen.

Förderung Der vorliegende Artikel ist entstanden im Rahmen des von der DFG geförderten Projekts „Das Imaginäre an den Grenzen des Sozialen“ (Projektnummer: 417783052).

Funding Open Access funding enabled and organized by Projekt DEAL.

Open Access Dieser Artikel wird unter der Creative Commons Namensnennung 4.0 International Lizenz veröffentlicht, welche die Nutzung, Vervielfältigung, Bearbeitung, Verbreitung und Wiedergabe in jeglichem Medium und Format erlaubt, sofern Sie den/die ursprünglichen Autor(en) und die Quelle ordnungsgemäß nennen, einen Link zur Creative Commons Lizenz beifügen und angeben, ob Änderungen vorgenommen wurden.

Die in diesem Artikel enthaltenen Bilder und sonstiges Drittmaterial unterliegen ebenfalls der genannten Creative Commons Lizenz, sofern sich aus der Abbildungslegende nichts anderes ergibt. Sofern das betreffende Material nicht unter der genannten Creative Commons Lizenz steht und die betreffende Handlung nicht nach gesetzlichen Vorschriften erlaubt ist, ist für die oben aufgeführten Weiterverwendungen des Materials die Einwilligung des jeweiligen Rechteinhabers einzuholen.

Weitere Details zur Lizenz entnehmen Sie bitte der Lizenzinformation auf http://creativecommons.org/ licenses/by/4.0/deed.de.

\section{Literatur}

Adams, Suzi, Paul Blokker, Natalie Doyle, John Krummel, und Jeremy Smith. 2015. Social imaginaries in debate. Social Imaginaries 1:15-52.

Akremi, Leila. 2016. Kommunikative Konstruktion von Zukunftsängsten: Imaginationen zukünftiger Identitäten im dystopischen Spielfilm. Wiesbaden: Springer VS.

Anderson, Benedict. 1991. Imagined communities: reflections on the origin and spread of nationalism. London: Verso.

Bataille, Georges. 1972. Das obszöne Werk. Reinbek: Rowohlt.

Bataille, Georges. 1999. Abjection and miserable forms. In More \& less, Hrsg. Sylvère Lotringer und Chris Kraus, 8-13. Cambridge: MIT Press.

Beckert, Jens. 2018. Imaginierte Zukunft. Fiktionale Erwartungen und die Dynamik des Kapitalismus. Berlin: Suhrkamp.

Behuniak, Susan M. 2011. The living dead? The construction of people with Alzheimer's disease as zombies. Ageing \& Society 31:70-92.

Biebert, Martina F., und Michael T. Schetsche. 2016. Theorie kultureller Abjekte. Zum gesellschaftlichen Umgang mit dauerhaft unintegrierbarem Wissen. Behemoth 9:97-123.

Bielefeldt, Heiner. 2016. Menschenwürde und Autonomie am Lebensende. Perspektiven der internationalen Menschenrechte. In Autonomie und Menschenrechte am Lebensende, Hrsg. Caroline Welsch, Christoph Ostgathe, Andreas Frewer, und Heiner Bielefeldt, 45-66. Bielefeld: transcript.

Binder, Werner. 2016. Magma und Scholle. Das soziale Imaginäre und die Wissenssoziologie. In Wissensforschung - Forschungswissen, Hrsg. Jürgen Raab und Reiner Keller, 533-543. Weinheim, Basel: Beltz Juventa.

Bourdieu, Pierre. 2005. Die männliche Herrschaft. Frankfurt a. Main: Suhrkamp.

Brauer, Kai, Frank Adloff, und Larissa Pfaller. 2014. „Wie mit mir umzugehen ist“. Zur biographischen Relevanz und Prospektivität von Patientenverfügungen. Soziale Welt 65:425-449.

Bröckling, Ulrich, Christian Dries, Matthias Leanza, und Tobias Schlechtriemen (Hrsg.). 2015a. Das Andere der Ordnung. Theorien des Exzeptionellen. Weilerswist: Velbrück Wissenschaft.

Bröckling, Ulrich, Christian Dries, Matthias Leanza, und Tobias Schlechtriemen. 2015b. Das Andere der Ordnung denken. Eine Perspektivverschiebung. In Das Andere der Ordnung. Theorien des Exzeptionellen, Hrsg. Ulrich Bröckling, Christian Dries, Matthias Leanza, und Tobias Schlechtriemen, 9-52. Weilerswist: Velbrück Wissenschaft.

Bröckling, Ulrich, Christian Dries, Matthias Leanza, und Tobias Schlechtriemen. 2018. Out of order Soziologie jenseits der Ordnungsbias. Skizze eines Forschungsprogramms. In Heterodoxie. Konzepte, 
Traditionen, Figuren der Abweichung, Hrsg. Michael Schetsche und Ina Schmied-Kittel, 255-270. Köln: Herbert von Halem.

Bührmann, Andrea D., und Werner Schneider. 2008. Mehr als nur diskursive Praxis? konzeptionelle Grundlagen und methodische Aspekte der Dispositivanalyse. Historical Social Research 33:108-141.

Bülow, Morten Hillgaard, und Marie-Luise Holm. 2016. Queering "successful ageing", dementia and alzheimer's research. Body \& Society 22:77-102.

Butler, Judith. 1997. Körper von Gewicht: die diskursiven Grenzen des Geschlechts. Frankfurt a. Main: Suhrkamp.

Castoriadis, Cornelius. 1984. Gesellschaft als imaginäre Institution. Entwurf einer politischen Philosophie. Frankfurt a. Main: Suhrkamp.

Castoriadis, Cornelius. 2010. Das Imaginäre: die Schöpfung im gesellschaftlich-geschichtlichen Bereich. In Das imaginäre Element und die menschliche Schöpfung, Hrsg. Cornelius Castoriadis, 25-45. Lich: Edition AV. Ausgewählte Schriften 3.

Castoriadis, Cornelius. 2012. Psychische Monade und Autonomes Subjekt. Lich: Edition AV. Ausgewählte Schriften 5 .

Delitz, Heike. 2019. Theorien des gesellschaftlichen Imaginären. Österreichische Zeitschrift für Soziologie 44:77-98.

Durkheim, Emile. 1994. Die elementaren Formen des religiösen Lebens. Frankfurt a. Main: Suhrkamp.

van Dyk, Silke. 2016. The othering of old age: insights from postcolonial studies. Journal of Aging Studies 39:109-120.

van Dyk, Silke, und Stephan Lessenich. 2009. Die jungen Alten. Analyse einer neuen Sozialfigur. Frankfurt a. Main: Campus.

Emirbayer, Mustafa, und Ann Mische. 1998. What Is Agency? American Journal of Sociology 103:9621023.

Fourastié, Brigitte, und Philippe Joron. 1993. The imaginary as a sociological perspective. Current Socio$\log$ 41:53-58.

Gee, Ellen, und Gloria Gutman (Hrsg.). 2000. The overselling of population ageing: apocalyptic demography, Intergenerational challenges, and social policy. Oxford, New York: Oxford University Press.

Gertenbach, Lars. 2011. Cornelius Castoriadis: Gesellschaftliche Praxis und radikale Imagination. In Kultur. Theorien der Gegenwart, Hrsg. Stephan Moebius und Dirk Quadflieg, 277-289. Wiesbaden: VS.

Giesen, Bernhard. 2010. Zwischenlagen: das Außerordentliche als Grund der sozialen Wirklichkeit. Weilerswist: Velbrück.

Gilleard, Chris. 2017. From collective representations to social imaginaries: How society represents itself to itself. European Journal of Cultural and Political Sociology 4:1-21. online first.

Gilleard, Chris, und Paul Higgs. 2010. Aging without agency: theorizing the fourth age. Aging \& Mental Health 14:121-128.

Gilleard, Chris, und Paul Higgs. 2011. Ageing abjection and embodiment in the fourth age. Journal of Aging Studies 25:135-142.

Gilleard, Chris, und Paul Higgs. 2013. The fourth age and the concept of a ,social imaginary“: a theoretical excursus. Journal of Aging Studies 27:368-376.

Gilleard, Chris, und Paul Higgs. 2015. Social death and the moral identity of the fourth age. Contemporary Social Science 10:262-271.

Gronemeyer, Reimer. 2004. Die Alzheimer-Gesellschaft. Über die Barbarisierung des Alters. In In einem reichen Land. Zeugnisse alltäglichen Leidens an der Gesellschaft, Hrsg. Günter Grass, Daniela Dahn, und Johano Strasser, 376-384. München: Deutscher Taschenbuchverlag.

Härpfer, Claudius. 2016. Das relativ Imaginäre? Spurensuche zwischen Völkerpsychologie und Soziologie. In Wissensforschung - Forschungswissen, Hrsg. Jürgen Raab und Reiner Keller, 555-554. Weinheim, Basel: Beltz Juventa.

Herbrik, Regine. 2011. Die kommunikative Konstruktion imaginärer Welten. Wiesbaden: VS.

Herbrik, Regine. 2013. Das Imaginäre in der (Wissens-)Soziologie und seine kommunikative Konstruktion in der empirischen Praxis. In Kommunikativer Konstruktivismus. Wissen, Kommunikation und Gesellschaft, Hrsg. Reiner Keller, Jo Reichertz, und Hubert Knoblauch, 295-315. Wiesbaden: Springer VS.

Herbrik, Regine, und Tobias Schlechtriemen. 2016. Das „Soziale Imaginäre“ der Gesellschaft und der Wissenssoziologie. Zur Einleitung. In Wissensforschung - Forschungswissen, Hrsg. Jürgen Raab und Reiner Keller, 531-532. Weinheim, Basel: Beltz Juventa.

Herbrik, Regine, und Tobias Schlechtriemen. 2019. Editorial for the special issue "Scopes of the Social Imaginary in Sociology" in the ÖZS. Österreichische Zeitschrift für Soziologie 44:1-15.

Higgs, Paul, und Chris Gilleard. 2014. Frailty, abjection and the „othering“ of the fourth age. Health Sociology Review 23:10-19. 
Higgs, Paul, und Chris Gilleard. 2017. The nature of the fourth age as a challenge to aging societies. In Planning later life. Bioethics and public health in aging societies, Hrsg. Mark Schweda, Larissa Pfaller, Kai Brauer, Frank Adloff, und Silke Schicktanz, 57-72. London, New York: Routledge.

Hövelmeyer, Marion. 2007. Pandoras Büchse. Konfigurationen von Körper und Kreativität. Bielefeld: transcript.

Katz, Stephen. 1992. Alarmist demography: power, knowledge, and the elderly population. Journal of Aging Studies 6:203-225.

Katz, Stephen, und Peter Whitehouse. 2017. Legacies, generations, and ageing futures. The ethics of intergenerativity. In Planning later life. Bioethics and public health in aging societies, Hrsg. Mark Schweda, Larissa Pfaller, Kai Brauer, Frank Adloff, und Silke Schicktanz, 240-253. London, New York: Routledge.

Knaut, Annette. 2015. Soziologische Imaginative - Der Begriff der Öffentlichkeit. In Routinen der Krise - Krise der Routinen. Verhandlungen des 37. Kongresses der Deutschen Gesellschaft für Soziologie, Trier, 2014, Hrsg. Stephan Lessenich, 1384-1394. http://publikationen.soziologie.de/index.php/ kongressband_2014. Zugegriffen: 23.03.2021.

Kristeva, Julia. 1982. Powers of horror: an essay on abjection. New York: Columbia University Press.

Lacan, J. 2001. Ecrits: a selection. London: Routledge.

Lamont, Michéle. 1992. Money, morals, and manners. The culture of the French and the American uppermiddle class. Chicago: University of Chicago Press.

Laslett, Peter. 1989. A fresh map of life: the emergence of the third age. London: Weidenfeld and Nicolson.

Lindemann, Gesa. 2002. Die Grenzen des Sozialen. Zur sozio-technischen Konstruktion von Leben und Tod in der Intensivmedizin. München: Fink.

Lindemann, Gesa. 2009. Das Soziale von seinen Grenzen her denken. Weilerswist: Velbrück.

Lindemann, Gesa. 2011. Anthropologie, gesellschaftliche Grenzregime und die Grenzen des Personseins. Ethik in der Medizin 23:35-41.

Maffesoli, Michel. 1993a. Introduction. Current Sociology 41:1-5.

Maffesoli, Michel. 1993b. The imaginary and the sacred in Durkheim's sociology. Current Sociology 41:59-67.

Mannheim, Karl. 1964. Wissenssoziologie. Auswahl aus dem Werk. Berlin, Neuwied: Luchterhand.

Mannheim, Karl. 1965. Ideologie und Utopie. Frankfurt a. Main: Schulte-Bulmke.

Mead, George Herbert. 1973. Geist, Identität und Gesellschaft aus der Sicht des Sozialbehaviorismus. Frankfurt a. Main: Suhrkamp.

Meier, Matthias. 2015. Relikte der Strata. Wiesbaden: Springer VS.

Menninghaus, Winfried. 2003. Disgust: theory and history of a strong sensation. Albany: SUNY Press.

Moscovici, Serge. 2000. Social representations: explorations in social psychology. New York: Wiley.

Niekriez, Yvonne. 2011. Gemeinschaft als Metapher. Das Imaginäre als Ordnungsschema. In Metaphern und Gesellschaft, Hrsg. Matthias Junge, 15-30. Wiesbaden: VS.

Pfaller, Larissa, und Mark Schweda. 2017. „Successful Aging“ und gutes Altern. Zur Reflexion gerontologischer Leitbilder. Angewandte GERONTOLOGIE Appliquée 17:20-21.

Schicktanz, Silke. 2017. The visionary shaping of dementia research. Imaginations and scenarios in biopolitical narratives and ethical reflections. In Planning later life. Bioethics and public health in aging societies, Hrsg. Mark Schweda, Larissa Pfaller, Kai Brauer, Frank Adloff, und Silke Schicktanz, 205-227. London, New York: Routledge.

Schlechtriemen, Tobias. 2014. Bilder des Sozialen. Das Netzwerk in der soziologischen Theorie. Paderborn: Wilhelm Fink.

Schmitz, Bettina. 2000. Die Unterwelt bewegen: Politik, Psychoanalyse und Kunst in der Philosophie Julia Kristevas. Aachen: ein-FACH-verlag.

Schroeter, Klaus R. 2004. Zur Doxa des sozialgerontologischen Feldes: Erfolgreiches und produktives Altern - Orthodoxie, Heterodoxie oder Allodoxie? Zeitschrift für Gerontologie und Geriatrie 37:51-55.

Schünzel, Anja. 2019. „Thinspire me“- Zur Bedeutung des sozialen Imaginären in Pro-Ana. Österreichische Zeitschrift für Soziologie 44:179-193.

Schütz, Alfred. 1971. Das Wählen zwischen Handlungsentwürfen. In Gesammelte Aufsätze, Bd. 1, 77-110. Den Haag: Martinus Nijhoff.

Schütz, Alfred, und Thomas Luckmann. 2003. Strukturen der Lebenswelt. Konstanz: UVK.

Schwenk, Jochen. 2019. Soziales Imaginäres und Stadtforschung. Österreichische Zeitschrift für Soziologie 44:99-115.

Shildrick, Margrit. 2001. Embodying the monster: encounters with the vulnerable self. London, Thousand Oaks, New Delhi: SAGE. 
Simmel, Georg. 1992. Soziologie. Untersuchungen über die Formen der Vergesellschaftung. Georg Simmel Gesamtausgabe, Bd. 11. Frankfurt a. Main: Suhrkamp.

Swinnen, Aagje, und Mark Schweda (Hrsg.). 2015. Popularizing dementia: public expressions and representations of forgetfulness. Bielefeld: transcript.

Tappenbeck, Inka. 1999. Phantasie und Gesellschaft: zur soziologischen Relevanz der Einbildungskraft. Würzburg: Königshausen \& Neumann.

Taylor, Charles. 2002. Modern social Imaginaries. Public Culture 14:91-124.

Taylor, Charles. 2004. Modern social Imaginaries. Durham: Duke University Press.

The Social Imaginaries Editorial Collective. 2015. Editorial. Social Imaginaries 1:7-13.

van Wijngaarden, Els, Carlo Leget, Anne Goossensen, Robert Pool, und Anne-Mei The. 2017. A captive, a wreck, a piece of dirt: aging anxieties embodied in older people with a death wish. Omega https:// doi.org/10.1177/0030222817732465.

Völk, Malte. 2019. Completing a meeting. Fabula 60:354-371.

Watier, Patrick. 1993. Simmel and the image of individuality. Current Sociology 41:69-75.

Wolf, Harald (Hrsg.). 2012. Das Imaginäre im Sozialen. Zur Sozialtheorie von Cornelius Castoriadis. Göttingen: Wallstein.

Zeilig, Hannah. 2014. Dementia as a cultural metaphor. The Gerontologist 54:258-267.

Larissa Pfaller Dr. Larissa Pfaller ist wissenschaftliche Mitarbeiterin am Institut für Soziologie der FAU Erlangen-Nürnberg. Ihre Schwerpunkte liegen in der rekonstruktiven Sozialforschung sowie der Kulturund Wissenssoziologie. Seit 2019 leitet sie das DFG-Projekt „Das Imaginäre an den Grenzen des Sozialen“, in dessen Kontext der vorliegende Artikel entstanden ist. 